\title{
REASONS FOR OMISSION OF PHYSIOTHERAPY IN A TERTIARY CARE INSTITUTE IN MUMBAI
}

\author{
Sanjith Saseedharan ${ }^{* 1}$, Paulomi Shah ${ }^{2}$, Vaijayanti Kadam ${ }^{3}$.
}

${ }^{*}$ Head-Department of Critical Care, S.L. Raheja Hospital (A Fortis Associate), Mumbai, Maharashtra, India.

${ }^{2}$ Registrar, Dept of Critical Care, S. L. Raheja hospital (A Fortis Associate), Mumbai, Maharashtra, India.

${ }^{3}$ Associate Consultant, Dept of Critical Care, S. L. Raheja hospital (A Fortis Associate), Mumbai, Maharashtra, India.

\section{ABSTRACT}

Background: As critical care is progressing, we are able salvage many patients, many of which in the past decades, would not have survived. A good number of these patients spend prolonged time in the intensive care unit(ICU). The after effect of which is myopathy, respiratory muscle weakness, neuropathy etc. Physiotherapy is an especially important armamentarium in the arsenal of critical care medicine which helps in the prevention of morbidity. However, there are reasons why there is omission in the performance of physiotherapy in the intensive care unit.

Purpose of study: To study the commonest reasons of omission of physiotherapy in a tertiary care hospital intensive care unit and thus help in devising methods or intervention for prevention of the same.

Methods: This study was performed by reviewing data from the charts of 240 consecutive patients admitted to a mixed medical surgical intensive care unit in Mumbai. Patients were prescribed physiotherapy by the intensive care physician a day in advance depending on the clinical indications and suitability for the same. The physiotherapist then decided the therapy for the following days and scheduled them accordingly. The reason of the omission of the session was documented by the physiotherapist responsible for the conduct of the session. The common reasons for omission of sessions were noted for analysis.

Results: Physiotherapy was missed in $32 \%$ of cases and this was predominantly seen in the morning hours. The most common reason (36\%) was patient refusal, attributed to inadequate sleep in the night hours. The next common reason (22\%) was the requirement of shifting the patient to the diagnostic room for investigations, namely imaging, and hence omission of physiotherapy. This was followed by bedside rounds by senior consultants including teaching rounds (18\%) followed by the sudden deterioration of condition in $16 \%$ of cases.

Conclusions: There are many obstacles that come in the way of the conduct of physiotherapy in large tertiary care busy intensive care units which is compounded with the lack of manpower. Like any medication assessment for the need for physiotherapy, timing of physiotherapy, the dose of physiotherapy and the actual form of therapy is a very important in improving patient care. Simple interventions can be put in place in order to avoid omission of physiotherapy.

KEY WORDS: Physiotherapy, Intensive care unit, Omission.

Address for correspondence: Dr. Sanjith Saseedharan, Dept of critical care,S.L.Raheja Hospital (A Fortis associate), Raheja Marg,Police colony, Mahim,Mumbai, Maharashtra, India. Phone number: 02266529999,9930925503,9004479549 E-Mail: docsanjith@ rediffmail.com

Access this Article online

Quick Response code

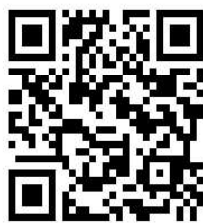

DOI: $10.16965 /$ ijpr.2020.166

Journal Information

International Journal of Physiotherapy and Research

ISSN (E) 2321-1822 | ISSN (P) 2321-8975

https://www.ijmhr.org/ijpr.htm

DOI-Prefix: https://dx.doi.org/10.16965/ijpr

\section{Article Information}

Received: 23 Aug 2020

Peer Review: 24 Aug 2020

Revised: 18 Sep 2020
Accepted: 29 Sep 2020

Published (O): 11 Oct 2020

Published (P): 11 Oct 2020 


\section{INTRODUCTION}

As intensive care research and development is increasing, we are able to salvage many more patients after long drawn fights with death in the critical care unit. It is not only important to save a patient but equally important to give patient a good quality of life. Physiotherapy is an important intervention that prevents and mitigates adverse effects of prolonged bed rest and mechanical ventilation during critical illness. Physiotherapy is incorporated as an integral part of management in intensive care in many countries. Many studies have reported reduced length of stay and improved outcomes[1,2].

In a systematic review of 10 randomized controlled trials it was shown that physiotherapy had no effect on mortality but reduced length of hospital and ICU stay and increased number of ventilator free days[3]. However in a recent metanalysis of 5 randomized controlled trials with a sample size of 603 patient it was shown that multimodal physiotherapy lowered mortality with no effect on length of stay. However, it is important to note that all the trials enrolled very few numbers of patients[4]. Hence there is a definite need for high quality trials and new evidence in this regards. Even the appropriate timing, nature of interventions and intensity ("dose") of physiotherapy remains unproven.

However, the prevention of this physical deconditioning and thus reduction in length of mechanical ventilation and stay in the intensive care unit will help in reducing cost. In most hospitals in developed countries, physiotherapy is seen as an integral part of the management of patients in ICUs. The precise role that physiotherapists play in the ICU varies considerably from one place to other depending on where ICU is located, local tradition, staffing levels, training, and expertise. The referral process is one example of this variation, whereby in some ICUs, physiotherapists assess all patients, whereas in other ICUs, patients are seen only after referral from medical staff[5].

There is no study from India that has looked into the common causes of omission of physiotherapy in indicated patients. The authors thus go on to find out reasons as to why physiotherapy was omitted and thus take probable measures to prevent it.

\section{MATERIALS AND METHODS}

This study involved data collection from 240 patients admitted to a 30 bedded mixed medical and surgical ICU over a period of 3 months at S.L Raheja Hospital, Mahim, Mumbai, Maharashtra. Two dedicated physiotherapists were appointed for managing the daily physiotherapy routine for patients prescribed physiotherapy in the intensive care unit. The intensive care physician was responsible for the prescription of physiotherapy in the unit. Each physiotherapy session spanned anywhere between 20 to 30 minutes depending on the illness and prescription. This included assessments for initiation of early mobilization and permissible activity levels by patient physiologic characteristics and diagnoses. The therapy included positioning, mobilization, manual hyperinflation, chest manipulation, suctioning, breathing, limb exercises and postural drainage. The physiotherapy sessions were planned a day in advance by designated physiotherapists based on patient diagnosis, requirement, improvement and deterioration in patients' condition. Same was followed on the day of session.

The sessions were divided into three slots, morning session 8am to $12 \mathrm{am}$, afternoon session 1 pm to $4 \mathrm{pm}$ and evening session $5 \mathrm{pm}$ to $8 \mathrm{pm}$. The average occupancy of the intensive care unit was $70 \%$ and at any given day there were not more than 10 patients for elective physiotherapy sessions. The physiotherapist formulated the exercise plans and timings for the concerned patients a day prior and created a timetable.

\section{Sample timetable:}

\begin{tabular}{|c|c|c|c|}
\hline Name & Morn shift & Afternoon shift & Evening shift \\
\hline$a b c$ & 8.00 am p1 & 1.00 pm p1 & 5.00 pm p1 \\
\hline$x y z$ & 8.00am p2 & 1.00 pm p2 & 5.00 pm p2 \\
\hline efg & $8.30 \mathrm{am}$ p1 & 1.30 pm p1 & 5.30 pm p1 \\
\hline Imn & $8.30 \mathrm{am} \mathrm{p} 2$ & 1.30 pm p2 & 5.30 pm p2 \\
\hline opq & $9.00 \mathrm{am}$ p1 & 2.00 pm p1 & 6.00 pm p1 \\
\hline rst & $9.00 \mathrm{am} \mathrm{p} 2$ & 2.00 pm p2 & 6.30 pm p2 \\
\hline uvw & $9.30 \mathrm{am}$ p1 & 2.30 pm p1 & 7.00 pm p1 \\
\hline jkl & 9.30 am p2 & 2.30 pm p2 & 7.30 pm p2 \\
\hline
\end{tabular}

P1-physiotherpist number 1

P2-physiotherpaist number 2

If for any reason the patient could not be provided with physiotherapy at the prescribed 
time(for e.g. $9.30 \mathrm{am}$ ) the patient was revisited in the designated shift time(for e.g. between 8 to $12.00 \mathrm{am}$ ) by either physiotherapist in order to complete the session.

The physiotherapist was provided with the following survey table which had to filled by the physiotherapist in case the session was omitted. These forms were then collected by the investigators and analyzed after the patient was discharged from the unit.

Table 2: Questionaries Performa.

\begin{tabular}{|c|c|}
\hline \multirow[t]{9}{*}{ Name of patient } & Shift- \\
\hline & Reasons for omission \\
\hline & 1.pt refusal - pain \\
\hline & - tired/not slept well \\
\hline & -others (e.g. irritability/not ok) \\
\hline & 2.visits to CT scan/U.S.G/M.R.I \\
\hline & $\begin{array}{l}\text { 3.interference with feeding cycle (just started or just } \\
\text { finished feeding) }\end{array}$ \\
\hline & 4.physician rounds \\
\hline & $\begin{array}{l}\text { 5.sudden change in patient condition that precludes } \\
\text { physiotherapy (for e.g.- atrial fibrillation, sudden cardiac } \\
\text { event) }\end{array}$ \\
\hline
\end{tabular}

Table 2 In case of physiotherapy session were missed the appropriate reason was tick marked by the physiotherapist. The common reasons included in the questionnaire were as per tab.2.

\section{RESULTS}

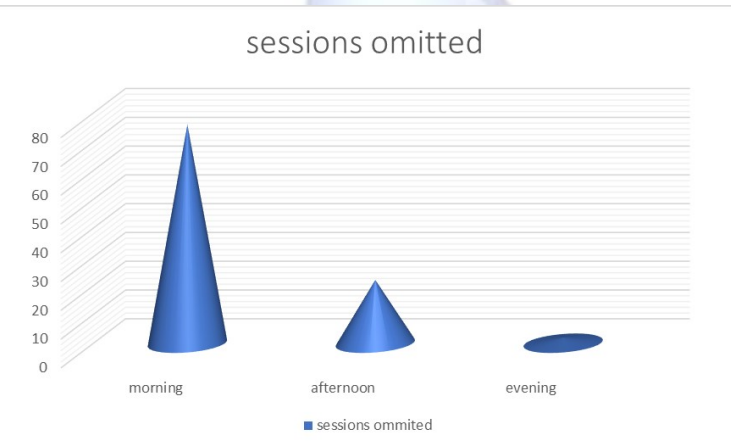

Fig. 1: Sessions omitted.

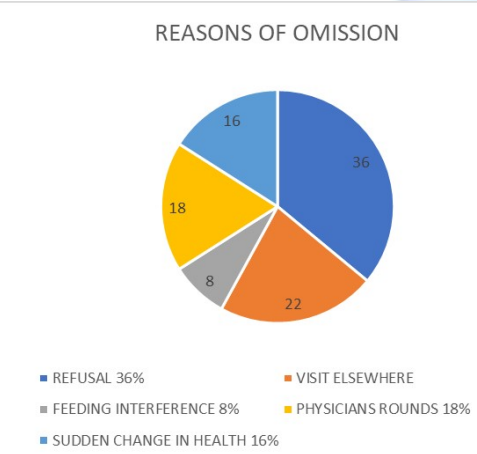

Fig. 2: Reasons of omission of physiotherapy.

The following results are drawn from the survey (Figure 1, Figure 2)

1. $32 \%$ of the patients were not able to receive physiotherapy on the scheduled timings.
2. Maximum hinderance to deliverance of physiotherapy was found during the morning shift.

\section{The reasons implicated were:}

A. Patient Refusal (36\%): Attributed to in adequate sleep the night prior,

accounting as the most common cause for delay or missing out on the physiotherapy session

B. Patient movement to alternate areas (22\%) Patients were shifted to scanning studies or subjected to imaging during the first half of the day

C. Feeding cycle related (8\%)- because the time coincided with meal timings in orally fed patients.

D. Daily rounds (18\%)- physician rounds, nursing rounds, bedside teaching

C. Unexpected change in vital parameters (16\%)-Sudden change in medical

condition especially heart rate and blood pressure and respiratory parameters

3. Afternoon physiotherapy (Post lunch sessions) sessions were missed out $22 \%$ of times. This was mostly related to delay in delivering food on proper timings

4. Evening physiotherapy was omitted only in $2 \%$ of cases and the reason being limited to sudden change in medical condition.

\section{DISCUSSION}

It is well understood that physiotherapy is an important intervention that prevents and mitigates adverse effects of prolonged bed rest and mechanical ventilation during critical illness. Rehabilitation delivered by the physiotherapist is tailored to patient needs and depends on conscious state, psychological status and physical strength. It incorporates any active and passive therapy that promotes movement and includes mobilization.

The National Institute for Health and Clinical Excellence (NICE), The European Respiratory Society and the European Society of Intensive Care Medicine recommend early assessment and management of physical morbidity (including mobilization and muscle training) delivered 
by physiotherapists and other health professionals. They also recommend that the physiotherapists should be responsible for implementing mobilization plans and exercise prescription in conjunction with other team members. Early mobilization can reduce ICU and hospital length of stay. A study that implemented a physiotherapy led early mobility protocol showed decreased intensive care unit and hospital length of stay ( 11.2 versus 14.5 days) and a potential cost saving of $7 \%$ of standard patient care costs

Even after understanding the potential advantages of physiotherapy in the icu this facet of therapy is not well studied. The authors by way of this study tried to understand the causes as to why physiotherapy was omitted in indicated patients.

This is the first Indian observational data from a large tertiary care intensive care unit to understand the reasons of omission of physiotherapy .

As per the results of this study it was noticed that among the sessions (i.e morning, afternoon and evening) the morning sessions involved maximum omissions.

The prime reasons for which was

A. Refusal as a result of improper sleep due to 1.noise level in the ICU at night hours (specifically nose from the monitors)

2.anxiety of having to sleep in an unaccustomed location and position.

\section{3.pain}

4.collection of labs at 4.00 am which disturbed all patients (this protocol was in place in the hospital so that laboratory results could be ready by 8:00 am)

5.Sponging of patient at 5.30 a.m. (which again disturbed the patient a second time and resulted in disturbed sleep)

B. Scheduled C.T. scans and MRI and planned procedures (with no information given to physiotherapists)

\section{Morning rounds of physicians}

A. Sudden change in the health for example an arrythmia like atrial fibrillation, supraventricular tachycardia or sinus tachycardia
There were very few omissions of physiotherapy in the afternoon and negligible omissions in evening hours sessions.

Physical therapy indeed is an important part of patient care. The above causes are "real world" reasons for omission of physiotherapy in our institute. Taking cue from the results of this study few protocol changes have been made in the author's institute which include

1. Reducing noise levels in the night by promptly addressing alarms.

2. Switching off lights in the nights so that patient get effective sleep.

3. Giving a small dose of injectable anxiolytic or oral anxiolytic so that patient gets adequate sleep in the night and remains fresh in the morning.

4. Scheduling blood collection and sponging together at $5.00 \mathrm{am}$ in the morning ensuring no more wakeup.

5. Early morning update to physiotherapist regarding scheduled scans so that the physiotherapy can be planned accordingly.

6. Completing rounds quickly and completely and doing bedside teaching in an adjoin room so that the physical therapy can continue.

7. Ensuring indication correction of electrolytes, oxygen level quickly in the morning avoiding arrhythmia like atrial fibrillation

8. Scheduling bronchodilators and cardiac medication 20 minutes prior to physiotherapy sessions to ensure smooth conduct

9. Co-ordinating diet timings with physiotherapy sessions so that there is minimal interruption of session.

\section{CONCLUSION}

Physiotherapy is an important part of the treatment of critically ill patients. However, there are many obstacles that lead to these therapy sessions not being conducted in time and effectively as shown in this paper. There is a great need to formulate country specific protocols in the assessment for the need for physiotherapy, timing of physiotherapy, the dose of physiotherapy and the actual form of therapy per session. There is also a need for large randomized control trials in the field of physiotherapy that could address the above issues. 


\section{ACKNOWLEDGEMENTS}

We acknowledge the physiotherapist Dr Reema and $\mathrm{Dr}$ Garima for helping the authors during data collection

\section{Conflicts of interest: None}

\section{REFERENCES}

[1]. Needham et al Early physical medicine and rehabilitation for patients with acute respiratory failure: a quality improvement project Arch Phys Med Rehabil 2010;91:536-542.

https://doi.org/10.1016/j.apmr.2010.01.002 PMid:20382284

[2]. Morris PE aet al. Early intensive care unit mobility therapy in the treatment of acute respiratory failure. Crit Care Med . 2008;36:2238-2243. https://doi.org/10.1097/CCM.0b013e318180b90e PMid:18596631

[3]. Kayambu G, Boots R, Paratz J. Physical therapy for the critically ill in the ICU: a systematic review and meta-analysis. Crit Care Med. 2013 Jun;41(6):154354. doi: 10.1097/CCM.0b013e31827ca637 https://doi.org/10.1097/CCM.0b013e31827ca637 PMid:23528802.
[4]. Pozuelo-Carrascosa DP, Torres-Costoso A, AlvarezBueno C, Cavero-Redondo I, López Muñoz P, MartínezVizcaíno V (2018) Multimodality respiratory physiotherapy reduces mortality but may not prevent ventilator-associated pneumonia or reduce length of stay in the intensive care unit: a systematic review. Journal of Physiotherapy 64: 222-228. https://doi.org/10.1016/j.jphys.2018.08.005 PMid:30220625

[5]. Herridge MS, Cheung AM, Tansey CM, et al. One-year outcomes in survivors of the acute respiratory distress syndrome. New England Journal of Medicine. 2003;348(8):683-93.

https://doi.org/10.1056/NEJMoa022450 PMid:12594312

How to cite this article:

Sanjith Saseedharan, Paulomi Shah, Vaijayanti Kadam. REASONS FOR OMISSION OF PHYSIOTHERAPY IN A TERTIARY CARE INSTITUTE IN MUMBAI. Int J Physiother Res 2020;8(5):3634-3638. DOI: 10.16965/ijpr.2020.166 